\title{
Numerical simulations of casing collapse: a case study in the South of Iran
}

\author{
R. Gholami ${ }^{1} \&$ V. Rasouli ${ }^{2}$ \\ ${ }^{I}$ Department of Mining, Petroleum and Geophysics, \\ Shahrood University of Technology, Iran \\ ${ }^{2}$ Department of Petroleum Engineering, Curtin University, Australia
}

\begin{abstract}
Collapse of casings during production has been reported on many occasions in different fields. Regardless of the root for the failure of the casings, this is due to stresses applied to the casing exceeding its ultimate strength. Failure of the casing similar to any pipe could be due to compressional, tensional, and burst or collapse forces due to various loads being applied to the casing string. Although several analytical models have been developed based on elastic or plastic approaches to estimate the ultimate strength of casing, it has been shown that these methods underestimate the casing strength. Casings' mechanical and geometrical parameters, effective in-situ stresses, temperature, formation properties and other intervening are simultaneously required for a complete design of casing. To simulate the failure of casing in the presence of various forces, numerical modeling is a robust approach that can be employed. In this study, finite element simulation was used through the use of ABAQUS software to model the failure of casings in one of the wells located in the southern part of Iran. The results revealed that increasing the diameter to thickness $(\mathrm{D} / \mathrm{t})$ ratio decreases the ultimate strength of casing, leading to unexpected failure in the wells under consideration. It was also shown that eccentricity drastically reduces the strength of casing. Since the numerical results of current study were in an acceptable agreement with experimental studies, numerical simulation method proposed here can be used to predict the casing collapse.

Keywords: numerical simulations, casing collapse, burst, collapse, axial force.
\end{abstract}




\section{Introduction}

Casing is a set of several steel pipes joined together and used to protect the wellbore after it is drilled. The casing is subjected to various loads in short term during drilling and long terms during production. Buckling due to axial load and burst and collapse as a result of high internal and external pressures, respectively, are examples of excessive loads and subsequent casing failure mechanisms [1]. Casing damage is perhaps one of the most frequently reported failures in oil and gas drilled wells [2]. This may happen during reservoir depletion due to excessive load caused by buckling or change in temperature gradient [3]. Casing failures induced by formation compaction have also been observed in various reservoirs located at the North Sea, the US Gulf of Mexico, California, South America and Asia [3]. These examples demonstrate that casing must be designed appropriately in order to resist excessive external forces during its intended life.

A casing with lower diameter to thickness ratio $(\mathrm{D} / \mathrm{t})$ and higher material strength will be more resistance to applied forces. However, optimum sizes should be chosen for economical purposes. To determine the proper casing specifications the worst loading condition that the casing may experience during its life should be identified. During installation, casing experiences a combination of pressure, bending and axial loads, but when it reaches to its predetermined location it would be under external pressure only. Practically, the hydrostatic pressure applied to the outside surface of casing inside the annulus space during drilling phase before cementing is perhaps the most important load to be considered for the design of casings.

Practical methods initially used for prediction of casing collapse were principally based on the empirical solutions. These equations have been developed to establish a linear or nonlinear relationship between collapse pressure and important parameters causing a casing to collapse [4-6]. From the reported literature the ratio of outside diameter to wall thickness $(\mathrm{D} / \mathrm{t})$, initial ovality and eccentricity are the most important geometrical parameters of the casing to be considered for design purposes. The important mechanical properties include the Young's modulus, Poisson's ratio and the yield strength of casing. Residual stress and applied axial stress are also needed to be considered.

Clinedinst [4] derived a theoretical formula accepted by the American Petroleum Institute (API) to predict casing collapse pressure based on assumption of elastic theory. This equation is presented as:

$$
P_{p}=2 \sigma_{y} \frac{(D / t-1)}{(D / t)^{2}}\left(1+\frac{1.47}{(D / t-1)}\right)
$$

where $P_{e}$ is the elastic pressure maintained by casing, $E$ is the Young's modulus, $v$ is the Poisson's ratio, $t$ is the wall thickness and $D$ is the diameter of casing.

Glock [7] proposed an empirical equation for hydrostatic buckling of confined elastic cylinders based on assumption of no friction between the ring and non-deformable medium, as well as no variation of stress and deformation in 
the axial direction of the cylinder. He assumed constant hoop force around the cylinder cross-section and proposed following equation:

$$
P_{e}=\frac{E}{\left(1-v^{2}\right)}\left(\frac{t}{D}\right)^{2.2}
$$

El-Sawy and Moore [8] found that the Glock equation can accurately predict the buckling pressure of elastic cylinders. However, it is important to realize that casing collapse occurs under plastic regime, as a result of which yield stress based on Von-Mises failure criterion has been embedded into the empirical equations. Further investigations have suggested that considering the yield stress through the Von-Mises criterion underestimates the collapse strength. Therefore, several equations were proposed later to predict the collapse of casing including both elastic and plastic behavior of the pipe [9-11].

Tamano et al. [9] proposed empirical equation evaluates the elasticplastic boundary of thick walled casing under external pressure as:

$$
P_{p}=2 \sigma_{y} \frac{(D / t-1)}{(D / t)^{2}}\left(1+\frac{1.47}{(D / t-1)}\right)
$$

In the above equation, $\sigma_{y}$ is the yield stress of casing.

Kyriakides and Youn [12] investigated buckling behavior of casing under external pressure using a semi-analytical formulation. The casing in that study was assumed to have an elastic-plastic behavior and showed to be accurate when it was used for collapse prediction of metal pipes [13]. The analytical equations proposed for estimation of collapse strength are limited to the assumptions used in their development and are reliable for specific type of materials. Numerical simulations may be used alternatively for such analysis.

In this study, an elastic-plastic FEM model was used to analysis the collapse strength of casing in one well located in South part of Iran. The results were validated through comparison with a series of experimental data obtained and presented by Madhavan [14].

\section{Study area}

The reservoir interval in this study is in Asmari formation. The oilfield approximately encompasses $441 \mathrm{~km}^{2}$ in the NE-SW direction. The average thickness of the reservoir is approximately $390 \mathrm{~m}$ in Asmari formation while the vertical thickness is nearly $790-830 \mathrm{~m}$ due to formation dipping. The initial oil in place of the formation is 43 MMMSTB and its initial pore pressure is $40 \mathrm{MPa}$ at the depth of $3350 \mathrm{~m}$. In the early years, mud weight with density of $1.15 \mathrm{gr} / \mathrm{cm}^{3}$ was used for the drilling, but it was reduced to $0.85 \mathrm{gr} / \mathrm{cm}^{3}$ in 2002 . The production from this reservoir through a number of drilled wells during past 50 years has led into a pore pressure to go down to approximately 9MPa. The current depletion rate is estimated to be $10-12 \mathrm{MPa}$ per year. The average porosity of the reservoir formation is approximately $12 \%$ in the Asmari formation [15]. This formation is mainly consisted of carbonate rocks but marl, shale and sandstone layers also exist. 
The field is producing for about 60 years now. The first casing collapse in this field was reported in one well in 1974. Since then, collapse of 48 wells drilled in the field has been reported indicating the need for further investigation of this problem. Figure 1 shows plot of number of wells experienced casing collapse each year. This figure shows that there is no trend indicating that the collapse has been due to the age of the pipe or bad cementing considering that similar casing pipes has been used and cementing jobs were done in a similar way in all wells. Past studies by many researchers suggest that there is no single mechanism responsible for the collapse of casing in this field but it due to combination of different mechanisms [2].

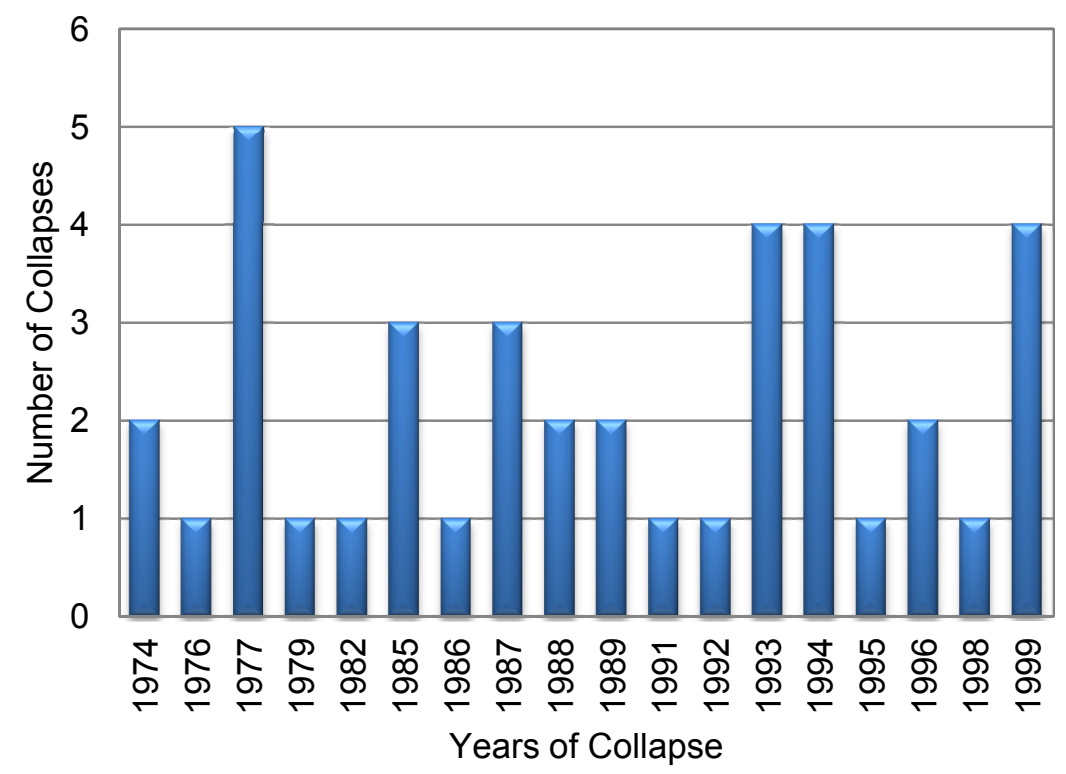

Figure 1: $\quad$ Casing collapse incidents during the history of the field [2].

\section{Finite element modelling}

In this study, general purpose finite-element software ABAQUS was numerically used to simulate the response of a casing under uniform external pressure using [16]. To analyses and simulate the buckling response of a pressurized casing, linear geometry and inelastic material behavior was considered for casing. It was assumed that there is no variation of loading and deformation in the longitudinal direction and hence, the simulations were carried out in two-dimensional (2D). This is what is generally called plane-strain conditions in which displacements in the longitudinal direction are restrained. This simplification has been reported to be valid since experimental studies proven that measured collapse pressure is independent of the casing length [1]. To prevent possible artificial locking in the 
calculation of stiffness matrices, a reduced integration technique was employed in the simulation [16]. Since the post- buckling shape of the cylinder would be symmetry, half of the cylinder cross-section was analyzed. To model the casing, four-node reduced integration shell elements (type S4R) were used, whereas eight- node brick elements (C3D8R) were used to simulate the surrounding medium. Figure 1 shows the typical finite-element mesh of the casing and elastic medium used in the present study. This dimension seems to be suitable for the surrounding medium since simulations of various model dimensions indicated a minor effect of larger domain and finer mesh on the accuracy of the modeling. The Von-Mises plasticity model along with isotropic hardening was considered to analysis the casing behavior under applied loads. It was found that kinematic hardening rule does not have an effect on the numerical modeling results. Along the contact areas between the cylinder and surrounded environment, a contact algorithm was taken into consideration to simulate the interface. For this purpose, a frictionless contact was assumed to take the effect of interface into account. At the end, a uniform external pressure was applied around the cylinder to examine its strength under various condition and pressure-deflection curves were plotted.

\section{Simulation results}

The casing response to the applied loads was studied for a range of $\mathrm{D} / \mathrm{t}$ ratios changing between 10 and 40 as these values are widely used for deep water applications in oil and gas wells. The material of the casing was assumed to be elastic with Young's Modulus of 200GPa and a Poisson's ratio of 0.3 while a frictionless interface was used to model the interface between the casing and the surrounding medium. The modulus of the medium was set equal to $20 \mathrm{GPa}$ (one tenth of the modulus of the casing [17]: this high value Young's Modulus ensures that the medium to be non-deformable and rigid during the analysis as it was observed from simulations. The simulation in ABAQUS started with applying an external pressure, which increase duo a maximum value and then decreased while a large deformation in the pipe was observed. The maximum pressure recorded corresponds to collapse pressure of the casing. Figure 1 shows the meshed geometry of the model before and after applying the external pressure.

The model shown in Figure 1 corresponds to a casing with D/t ratio of 10 which collapsed at an external pressure of 50MPa. At this time the casing experienced $3 \mathrm{~cm}$ displacement in Y-direction. The casing shows compression forces at $\theta=90^{\circ}$ (from $\mathrm{X}$ direction) whereas tensional forces apply to casing at an angle of $\theta=180^{\circ}$. Figure 2 and 3, respectively, shows the displacement magnitude and Von-Mises stress distribution inside a casing with D/t ratio of 10. 

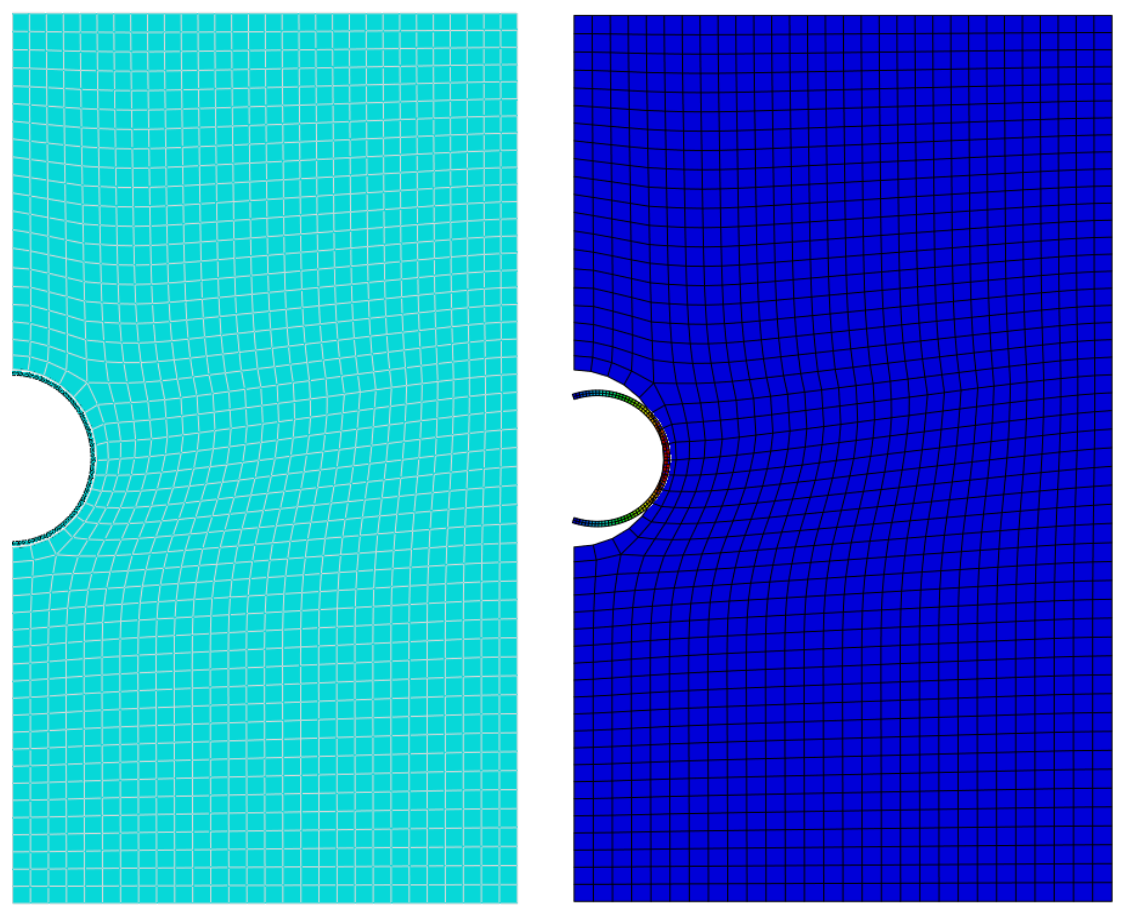

Figure 2: Model geometry before (left) and after (right) applying external uniform pressure.

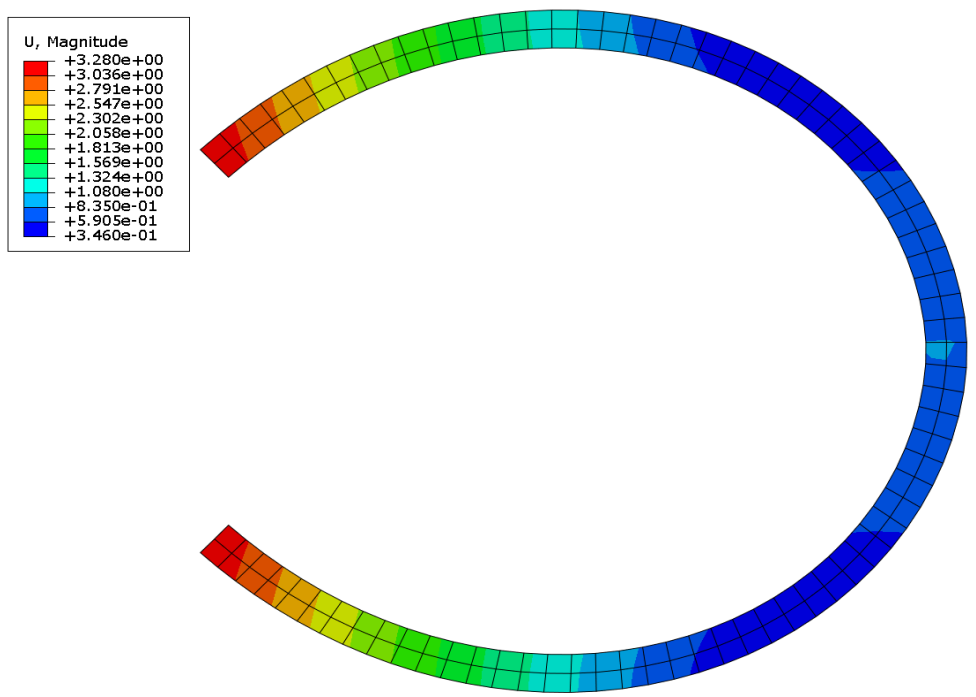

Figure 3: Displacement of casing wall at an external pressure of 50MPa. 


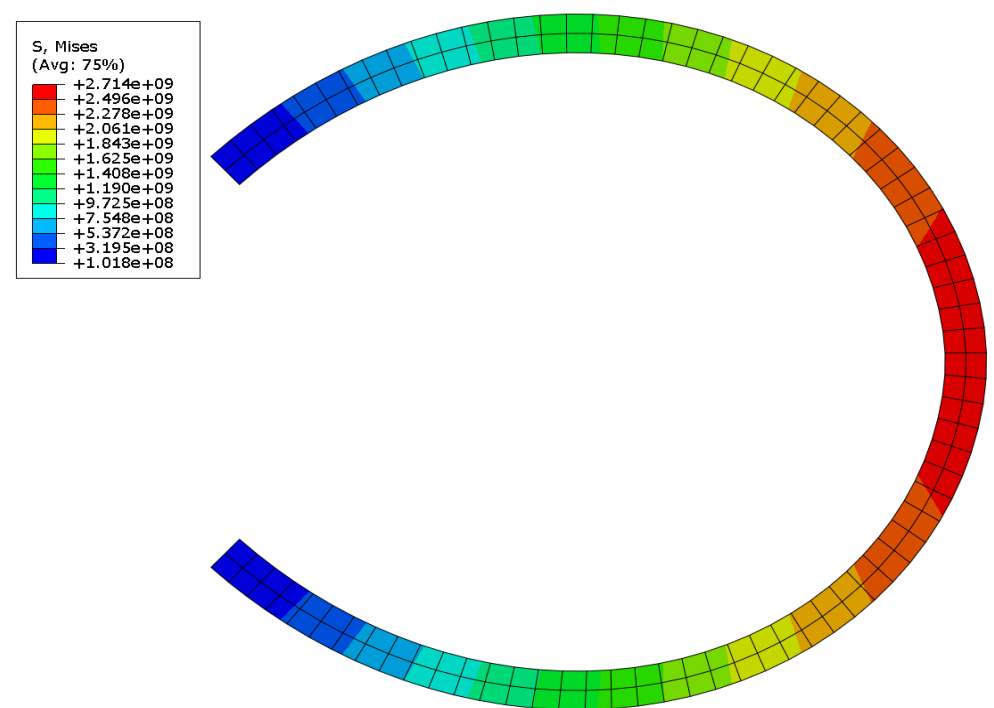

Figure 4: Von-Mises stress distribution of casing wall at an external pressure of $50 \mathrm{MPa}$.

To test the strength of casings with various $\mathrm{D} / \mathrm{t}$ ratios, four numerical models were built according to the procedure explained above and examined the amount of external pressure which can be sustained by the casings before they fail. Figure 4 gives the plot of external pressures versus displacement for casings with various $\mathrm{D} / \mathrm{t}$ ratios.

According to Figure 4, it is seen that decreasing the diameter to thickness ratio $(\mathrm{D} / \mathrm{t})$ significantly decreases the collapse resistance of casing. Similar results were obtained by $[1,3]$, demonstrating the validity of the results of presented numerical models in this study.

However, the results of experimental and analytical studies [18, 19] indicate that the geometric imperfections can drastically reduce the collapse pressures. The two types of geometric imperfections are ovality and eccentricity of casing. In this paper, the eccentricity effect on casing collapse was only studied. Information about ovality effect on casing collapse can be found in [20].

Since wall thickness of a casing varies along the line of cross-section, the term eccentricity is defined as [1]:

$$
\varepsilon=\frac{t_{\max }-t_{\min }}{t_{\max }+t_{\min }}
$$

The casing wall thickness is usually measured with a micrometer at approximately twelve circumferential points at each end of the test specimens. In this study, to assess the effect of initial eccentricity on the ultimate strength of casing, an eccentricity was introduced in the model by shifting the center of inner circle of the casing wall. The meshing and modeling process was to those 
casing with no eccentricity. The model was then subjected to external uniform pressure and the results were recorded. Figure 5 shows the Plastic Strain (PE) values computed for an imperfect casing wall after reaching the collapse pressure.

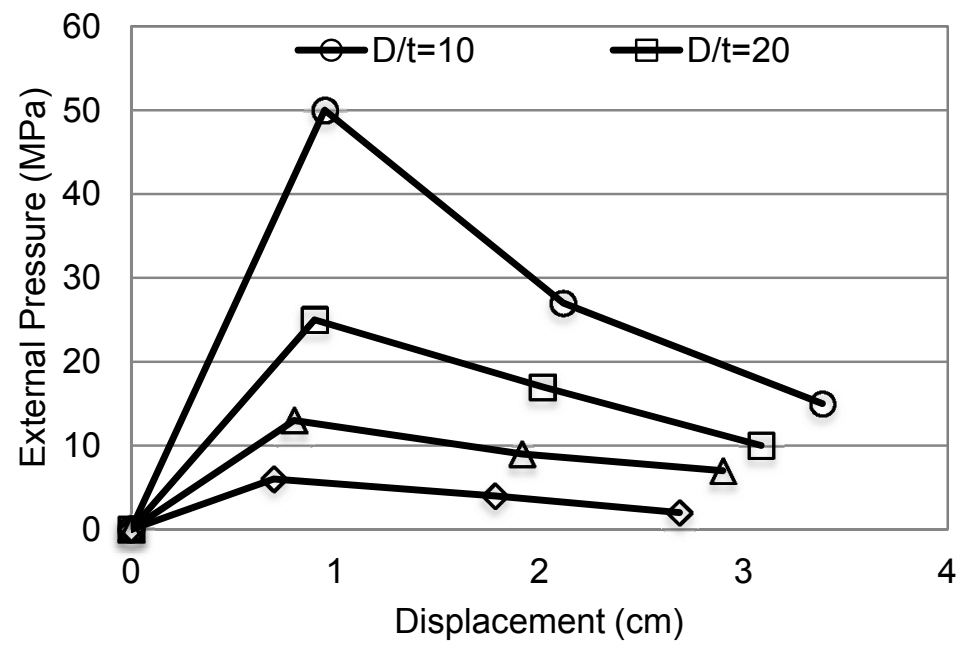

Figure 5: Comparison of collapse pressure in casings with different $\mathrm{D} / \mathrm{t}$ ratios.

Depicted in Figure 5, eccentricity decreases the strength of casing in the regions where diameter to thickness ratio reaches to its highest value. As it can be seen, the region with minimum thickness undergoes the maximum strain, demonstrating the effect of eccentricity on the collapse pressure. Huang et al. [1] pointed out that initial eccentricity causes reduction of strength of casing in regions with high $\mathrm{D} / \mathrm{t}$ values. To validate the numerical modeling procedure and results obtained during this study, a validation was performed utilizing the experimental data presented by Madhavan [14]. This is presented in the following section.

\section{Model validation}

The finite element analysis method proposed in this paper was validated by comparing against experimental data corresponding to steel casings used commercially and presented by Madhavan [14]. The seamless, cold drawn, 304 stainless steel casings which are manufactured according to the API Specification 5LX standards were used for comparison purposes in this study. This type of casing was chose since they are commercially available and has similarity to material behavior of typical pipeline grade steel. Casings are designed to resist against severe geological and environmental conditions, 
therefore different casing collapse data is presented with a variation of yield strength depending on specific application. In presented Tables the ratio of diameter to thickness of test specimens was between 10 and 40 . To measure the steel imperfection, the specimens were sealed at both ends and placed in a highpressure test facility. The collapse experiments were carried out under volume controlled loading conditions. The applied external pressure was monitored using a calibrated pressure transducer and analogue time-based recording equipment. The pressurization rate was low enough to avoid strain rate effects. The geometry and stiffness of casings used for experimental study were carefully imported into the ABAQUS numerical modeling program. Figure 6 compares the results of FEM and those of the experiments.
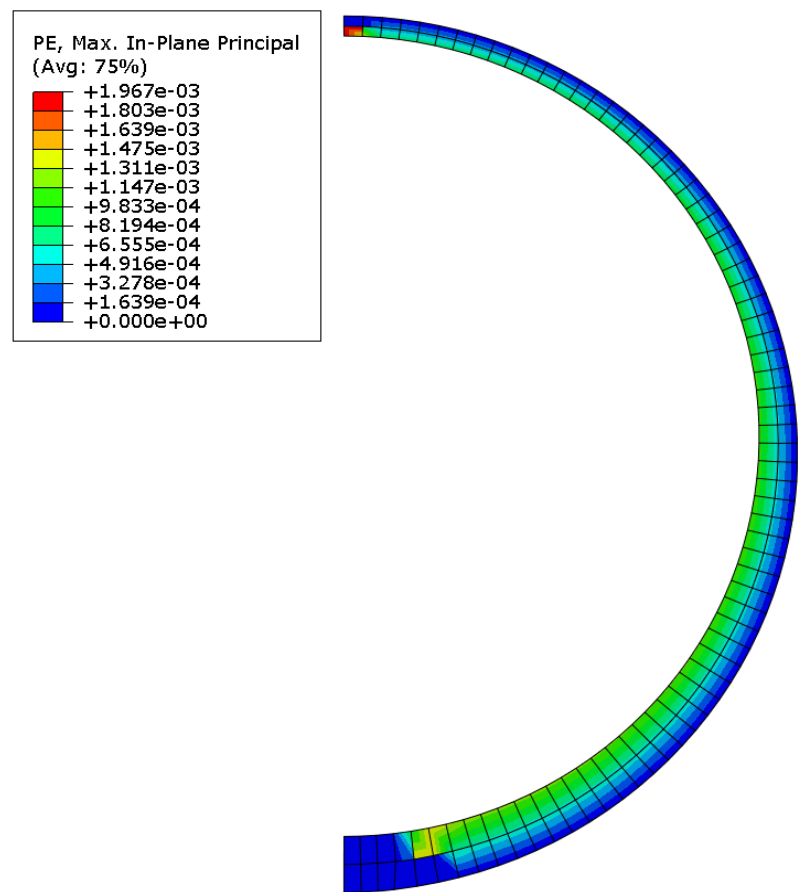

Figure 6: Plastic Strain (PE) values computed according to the external pressure applied on the outer sides of the casing.

As is shown in Figure 6, there is an acceptable agreement between experimental and numerical data with maximum deviation of $5 \%$, which is in an acceptable range.

Although the effects of diameter to thickness ratio as well as eccentricity were investigated on the strength and collapse pressure of casing, there are other parameters the need to be considered when modeling casing collapse. For example plastic formation or high permeability layers surrounding the casing can cause stress concentration and casing deformation [1]. Poor cementing job, 
poorly sealed casing, material anisotropy and initial ovality are other important factors which can significantly reduce the collapse strength of casing.

\section{Conclusions}

In this paper, numerical simulations were applied to evaluate the casing collapse strength. The data belongs to one of the wells in Southern part of Iran. The effect of casing eccentricity and ovality of casing were investigated and validated against experimental data. The results indicated that increasing the diameter to thickness ratio decreases the resistance of casing against external pressure. Eccentricity of casing indicated to decrease the strength of casing in regions where thickness has the lowest value. There are other parameters which can be considered in future studies for casing collapse modeling.

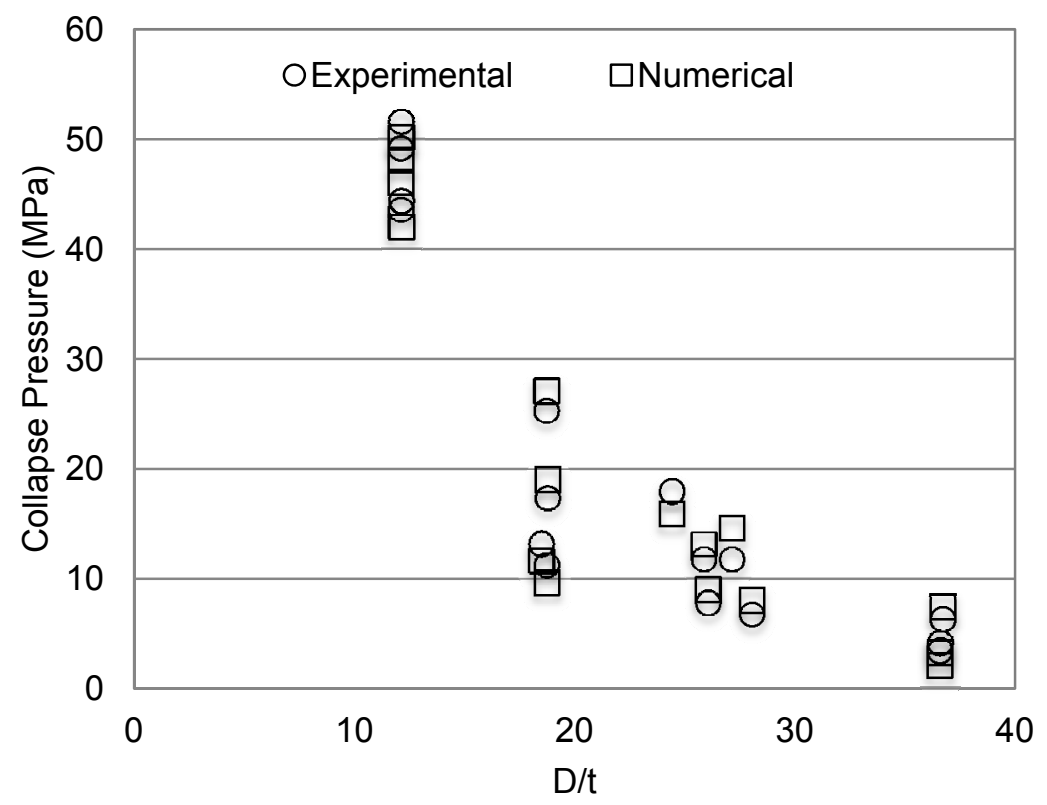

Figure 7: Comparison of numerical simulation results with experimental data.

\section{References}

[1] Huang, X., Mihsein, M., Kibble, K., Hall R. Collapse strength analysis of casing design using finite element method, International Journal of Pressure Vessels and Piping 77, 359-367, 2000. 
[2] Salehi, S., Hareland, G., Khademi Dehkordi, K., Ganji, M., Abdollahi, M. Casing collapse risk assessment and depth prediction with a neural network system approach, Journal of Petroleum Science and Engineering 69, 156162, 2009.

[3] Peng, S., Fu, J., Zhang, J. Borehole casing failure analysis in unconsolidated formations: A case study, Journal of Petroleum Science and Engineering 59, 226-238, 2007.

[4] Clinedinst WO. A rational expression for the critical collapsing pressure of pipe under external pressure. Drilling Production Practice, API: 383-391, 1939.

[5] Holmquist JL, Nadai A. A theoretical approach to the problem of collapse of deep well casing. Drilling Production Practice, API: 392-420, 1939.

[6] Timoshenko SP, Gere JM. Theory of elastic stability. 2nd ed. New York: McGraw-Hill, 1961.

[7] Glock D. Überkritisches Verhalten eines Starr Ummantelten Kreisrohres bei Wasserdrunck von Aussen und Temperaturdehnung. (Post-critical behavior of a rigidly encased circular pipe subject to external water pressure and thermal extension). Der Stahlbau; 7: 212-7, 1977.

[8] El-Sawy K, Moore ID. Stability of loosely fitted liners used to rehabilitate rigid pipes. Journal of Structural Engineering, ASCE; 124(11):1350-7, 1998.

[9] Tamano T, et al. A new empirical formula for collapse resistance of commercial casing. ASME Transactions of Energy Resources Technology: 489-495, 1983.

[10] Issa J. A. An improved design equation for tubular collapse. SPE 366 359367 26317, Proceedings of SPE Annual Technical Conference, Houston, Oct. 1993.

[11] API Bulletin 5C3 on Formulas and Calculations for Casing, Tubing, Drill Pipe and Line Pipe Properties. Fifth edition, July 1989.

[12] Kyriakides S, Youn SK. On the collapse of circular confined rings under external pressure. International Journal of Solids and Structures, 20(7): 699-713, 1984.

[13] Kyriakides S. Propagating buckles in long confined cylindrical shells. International Journal of Solids and Structures, 22(12):1579-97, 1986.

[14] Madhavan, R. On the collapse of a long Thick-walled circular tubes under biaxial loading, $\mathrm{PhD}$ thesis, California university of technology, 1988.

[15] NISOC, "NISOC R\&D Solutions Project \#1, casing collapse (well integrity); Phase 1-concept \& feasibility study, June 2005, Ahwaz, Iran.

[16] Hibbit HD, Karlsson BI, Sorensen P. Theory manual. ABAQUS, version 6.7, Providence, RI, USA, 2010.

[17] Boukani, S. Wellbore stability analysis for underbalanced drilling, MSc thesis, Petroleum University of Technology, Iran, 2006.

[18] Verner, E.A. and Langner, C.G., Collapse of Thick Wall Pipe in Ultra Deep Water, ASCE, Proceedings of Conference on Pipelines in Adverse Environments, San Diego, California, pp. 159-187, Nov. 1983. 
162 Petroleum and Mineral Resources

[19] Yeh, M.K. and Kyriakides, S., On the Collapse of Inelastic Thick-Walled Tubes Under External Pressure, ASME Journal of Energy Resources Technology, V. 108, Mar. 1986.

[20] Vasilikis, D., Karamanos, S.A. Stability of confined thin-walled steel cylinders under external pressure, International Journal of Mechanical Sciences 51, 21-32, 1988. 